\title{
Un locus autosomique prédisposant aux délétions de I'ADN mitochondrial
}

La chaîne respiratoire localisée dans la membrane interne des mitochondries produit la majeure partie de l'énergie cellulaire. Il n'est donc pas surprenant qu'une anomalie de cette chaîne provoque des troubles sérieux. Or les enzymes impliquées possèdent la particularité génétique de provenir de deux génomes physiquement séparés, celui du noyau et celui des mitochondries. On peut donc admettre en principe, qu'une anomalie due à une altération d'un gène nucléaire présente une hérédité mendélienne, et d'un gène mitochondrial une hérédité maternelle. On n'a encore identifié aucun gène nucléaire responsable d'un désordre de la chaîne respiratoire; en revanche, plusieurs maladies associées à des délétions de l'ADN mitochondrial (ADNmt) sont connues. On trouve également des mutations ponctuelles. Elles ont été décrites en 1988 et rapportées dans médecine/ sciences [1,2]. Mais, même s'il s'agit d'ADNmt, on conçoit que le noyau intervienne, puisqu'il règle la réplication et la transcription de l'ADNmt par l'intervention d'enzymes nécessaires à sa biosynthèse. En revanche, on ne connaît rien des interrelations entre les deux systèmes.

Un certain nombre de mystères restent à élucider dans les maladies du génome mitochondrial: par exemple, une même lésion moléculaire peut être à l'origine de tableaux cliniques très différents ; par ailleurs, on trouve, bien qu'en faible quantité, les mêmes délétions chez des sujets normaux au cours du vieillissement. Mais le plus surprenant reste la description, faite dès 1989 par une équipe italienne [3] et $\left(\mathrm{m} / \mathrm{s} n^{\circ} 2\right.$, vol. 7 p. 172), d'une maladie impliquant $\mathrm{m} / \mathrm{s} n^{\circ} 5$, vol. 11, mai 95 des délétions multiples de l'ADNmt, dont la transmission est autosomique dominante. La forme clinique était celle d'une ophtalmoplégie externe progressive (dite PEO), qui reste la plus courante, bien que d'autres aspects aient été décrits.

Jusqu'à présent aucun locus n'avait pu être incriminé comme responsable de ce type d'hérédité. Une équipe de Helsinki (Finlande) associée à des chercheurs français et italiens, vient de s'attaquer au problème [4]. Par crainte d'une hétérogénéité génétique qui obscurcirait les résultats, les auteurs ont préféré limiter les analyses de liaison à une seule famille de taille suffisante. Dans cette famille finnoise, les symptômes dominants sont l'ophtalmoplégie externe, un ptôsis, une intolérance à l'exercice. Le support anatomique est la présence des habituelles fibres musculaires rouges en lambeaux (ragged red (ibers). La maladie, dominante, a une pénétrance atteignant $100 \%$ après 50 ans. C'est dire que l'on trouve parmi les sujets jeunes des porteurs encore asymptomatiques qu'il est important de dépister. On peut les reconnaître sur des Southern blots qui montrent les délétions de l'ADNmt, mais il est indispensable d'obtenir une biopsie musculaire; on trouve, en effet, de grandes quantités d'ADNmt muté au niveau des muscles squelettiques alors que l'anomalie est très rarement présente sur l'ADNmt des leucocytes.

Dans un premier temps, l'analyse de 248 marqueurs répartis sur l'ensemble du génome a permis d'en récuser $70 \%$. Quand une liaison fut devenue apparente sur le bras long $\mathrm{du}$ chromosome 10 , on utilisa 11 marqueurs supplémentaires cou- vrant une région de $27 \mathrm{cM}$. Pour le marqueur le plus efficace, dit D10S597, on obtint un lod score de 4, 52 pour une fraction de recombinaison $\theta=0,0$. La comparaison avec deux gènes connus de la région, ( $H O X 11$ et $A D R B 1$, codant pour le récepteur adrénergique $\beta$-1) permit une localisation en $10 \mathrm{q} 23.3-24.3$. La comparaison avec deux familles italiennes, cliniquement semblables, montra l'hétérogénéité génétique de l'affection en excluant chez elles le locus en 10q. De nouveaux travaux seront donc nécessaires pour découvrir d'autres loci.

Les gènes qui pourraient être en cause dans les PEO seraient ceux qui interviennent dans la biogenèse des mitochondries. Leur connaissance est encore très incomplète. Mais on peut concevoir aussi une anomalie moins spécifique, par exemple des dommages causés à l'ADN par des radicaux libres réactifs, dont la production serait activée par des dysfonctionnements de la chaîne respiratoire.

Un certain nombre de gènes candidats peuvent être évoqués. Dans le cas de la famille finnoise, la localisation connue élimine par exemple la protéine mitochondriale liant l'ADN simple brin (gène en $7 q$ ) ; l'endonucléase $G(9 q)$, qui pourrait intervenir dans la transcription ou la réparation de l'ADNmt; les enzymes impliquées dans l'élimination des radicaux libres, (divers types de superoxyde dismutase, catalase, glutathion peroxydase), ont également des localisations différentes. Reste un candidat possible: c'est le gène codant pour le facteur de transcription mitochondrial, assigné à 10q [5]. Ce facteur est capable de plier ou de déplier l'ADN,

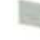
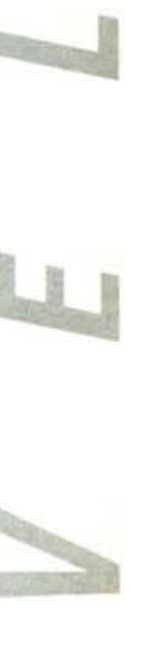
et peut-être aussi d'en régler le nombre de copies. Son gène a été cloné et séquencé. La comparaison de la partie codante entre un malade et un témoin n'a pas révélé de différences. Toutefois l'analyse n'a porté que sur un seul cas et n'a pas inclus les jonctions exons-introns ni les zones flanquantes. On ne saurait donc éliminer ce facteur, tout en n'ayant pas actuellement d'argument probant en sa faveur.

Les résultats obtenus à ce jour présentent déjà un intérêt considérable. La liaison génétique entre un locus situé sur un point précis d'un chromosome et des délétions multiples de l'ADNmt dans des PEO est la première démonstration d'une double implication génétique: une anomalie d'un gène nucléaire prédispose à des délétions de l'ADNmt. L'hétérogénéité génétique indique que d'autres gènes nucléaires peuvent être à l'origine du même type de délétions. La voie est ouverte pour l'identification complète de ces gènes, et un début de compréhension des interrelations entre noyau et mitochondries.

J.C.D.

1. Rōtig A, Bonnefond JP, Cormier V, Rustin P, Saudubray JM, Munnich A. Les remaniements du génome mitochondrial dans les déficits énergétiques de l'enfant: de nouvelles maladies de système: médecine/sciences $1989 ; 5: 459-71$.

2. Nelson 1, Degrel F, Marsac C, Ponsot G, Lestienne P. Des délétions de l'ADN mitochondrial dans le syndrome de Kearns-Sayre et autres myopathies avec ophtalmoplégie externe progressive. médecine/sciences $1989 ; 5: 472-9$.

. Zeviani M, Brisolin N, Gallera C, et al. An autosomal dominant disorder with multiple deletions of mitochondrial DNA starting at the D)-loop region. Nature 1989; 339 : 309-1 I

4. Suomalainen A, Kaukonen J, Amati l', Timonen R, Haltia M, Weissenbach J, Zeviani M Domer H, Peltonen I.. An autosomal locus predisposing to deletions of mitochondrial DNA. Nature Genet $1995 ; 9: 146.51$

5. Tiranti V, Rossi E, Ruiz-Carillo A, Rossi G, Rocchi M, DilDonato S, Zuffardi O, Zeviani M. Chromosomal localization of mitochondrial transcription factor $A$ (TCF6), single-stranded DNA-binding protein (SSBP), and endonuclease G (EN IOO(;), three human housekeeping genes involved in mitochondrial biogenesis. Genomics 1995; 25 : 559-64. 